\title{
Homogénéité des représentations dans l'entreprise : illusions et réalités
}

Arlette Bouzon

\section{(2) OpenEdition}

1 Journals

Édition électronique

URL : http://journals.openedition.org/communicationorganisation/2752

DOI : 10.4000/communicationorganisation. 2752

ISSN : $1775-3546$

Éditeur

Presses universitaires de Bordeaux

Édition imprimée

Date de publication : 1 novembre 2002

ISSN : 1168-5549

Référence électronique

Arlette Bouzon, «Homogénéité des représentations dans l'entreprise : illusions et réalités », Communication et organisation [En ligne], 22 | 2002, mis en ligne le 27 mars 2012, consulté le 19 avril 2019. URL : http://journals.openedition.org/communicationorganisation/2752 ; DOI : 10.4000/ communicationorganisation. 2752

Ce document a été généré automatiquement le 19 avril 2019

(c) Presses universitaires de Bordeaux 


\title{
Homogénéité des représentations dans l'entreprise : illusions et réalités
}

\author{
Arlette Bouzon
}

1 Au cours des années quatre-vingt, de nombreuses publications ont souligné les bienfaits apportés à l'entreprise par l'homogénéité du personnel, présentés comme étant susceptibles de rendre l'organisation plus efficace (Tapia, 1991). Cet engouement a été notamment illustré par le vif intérêt suscité par les travaux traitant de la «corporate culture » qui préconisaient une « culture forte » dans l'organisation (Bosche, 1984) et un large recouvrement des valeurs et des champs représentationnels des membres de celleci (Lemaître, 1984).

2 Bien que cette volonté d'uniformisation se soit quelque peu émoussée, une partie importante du management continue à promouvoir une entreprise homogène et consensuelle où toute trace de conflit aurait disparu (Le Goff. 1998) et où il serait possible d'infléchir le comportement des employés par diverses actions de communication et de gestion du personnel (Seignour. Dubois, 1999). Cette recherche d'une certaine cohésion, sinon dans les esprits, du moins dans les pratiques, peut sembler d'autant plus nécessaire aux dirigeants d'entreprises qu'elle s'opère aujourd'hui dans un environnement turbulent marqué par de nombreuses restructurations.

3 Mais quelle est la place des représentations individuelles dans le fonctionnement de l'entreprise ? L'uniformité renforce-t-elle la cohésion des acteurs et la cohérence de leurs actions, ou constitue-t-elle un frein au développement de l'esprit critique et à l'apparition d'idées nouvelles? En outre, cette influence supposée de l'homogénéité représentationnelle dans l'entreprise ne varie-t-elle pas selon les types d'activité ? Nous tenterons de répondre à ces questions en nous appuyant sur les résultats d'enquêtes de terrain que nous avons menées sur le sujet. 


\section{Vers une homogénéisation des représentations dans l'entreprise}

4 Pour améliorer l'efficacité de l'entreprise et assurer sa cohésion, certains dirigeants recherchent la conformité de leurs salariés à des normes qui leur sont propres. La coordination des actions engagées par ces différents acteurs ne leur semble en effet possible que si ces derniers partagent ensemble un langage, des connaissances, des modes de pensée et des valeurs similaires, ou du moins compatibles. Cette volonté peut notamment se traduire par le choix d'une politique de recrutement et de promotion interne ou par diverses pratiques de communications organisationnelles qui font parfois l'objet de phénomènes de mode.

5 Ainsi, la culture d'entreprise connut-elle un grand succès au début des années quatrevingt, après la publication d'ouvrages américains portant, entre autres, sur le management japonais (Ouchi, 1982), puis sur «l'excellence de la gestion» de certaines compagnies américaines (Peters, Waterman. 1983). Comparant le fonctionnement de diverses entreprises, ces ouvrages faisaient de cette "corporate culture" l'un des principaux facteurs identifiés de performance. L'enthousiasme suscité par ce nouveau «concept» peut notamment s'expliquer par le fait qu'essentiellement qualitatif, il s'opposait alors à la pensée dominante fondée exclusivement sur l'utilisation d'outils rationnels (structures, contrôles, ratios...), et associait les performances obtenues à des qualités subjectives: "l'adhésion, l'implication, et l'engagement du personnel» (D'Iribarne, 1986). Deux approches ont coexisté jusqu'au milieu des années quatre-vingt. La culture est d'abord conçue comme un élément de l'entreprise qui permet aux individus de s'adapter à l'organisation et à son environnement. Les auteurs de ce courant proposent de promouvoir la culture comme un moyen de canaliser, et de maîtriser, les valeurs et les comportements dans l'organisation. La seconde approche considère l'entreprise comme étant une culture, système de connaissances que chacun de ses membres peut interpréter par le biais de mécanismes mentaux. Cette deuxième conception offre notamment des pistes à l'audit de culture d'entreprise proposé par M. Thevenet (1985). La culture est partagée par les membres d'une entreprise. Elle est influencée par des variables culturelles externes et par des particularités propres à chaque organisation. Les variables culturelles externes sont composées de la culture nationale, celle du pays dans lequel est implantée l'entreprise et de ce qu'il est convenu d'appeler, sans connotation péjorative, les sous-cultures, c'est-à-dire les cultures propres à chaque groupe composant l'entreprise: origines régionales, sociales, ethniques du personnel, corps de métiers exercés dans l'entreprise... L'étude de l'histoire, des métiers, des valeurs, des signes et des symboles permet d'appréhender les grands traits culturels de l'entreprise.

6 Mais à côté de ces publications qui envisagent la culture comme un outil de gestion, d'autres écrits dénoncent l'emploi abusif de cette notion hors de l'anthropologie, son contexte d'origine, l'esprit manipulateur dont il relève et sa visée exclusivement instrumentale (Le Goff, 1998). Le succès rencontré auprès des milieux dirigeants par la culture d'entreprise s'explique d'ailleurs par l'apparente facilité à plaquer une «culture gagnante » à l'entreprise en lui faisant revêtir un véritable " prêt-à-porter symbolique ». Cependant après des expériences aux résultats souvent mitigés, cet intérêt pour la culture d'entreprise s'est aujourd'hui quelque peu estompé (Seignour, Dubois, 1999). 
7 Plus récemment, le « projet d'entreprise » fut proposé aux dirigeants pour constituer un manifeste des valeurs et objectifs de l'organisation. Mais, dans la pratique il se limita bien souvent à la rédaction d'un document rassemblant pêle-mêle des proclamations d'intentions et des valeurs (à) partagées, émanant de la sphère dirigeante. De plus, souvent inspirés par des cabinets-conseils ou des réalisations similaires déjà développées et diffusées dans la presse professionnelle, ces projets donnèrent parfois une impression d'uniformité et de « déjà vu », incompatible avec la spécificité de l'organisation. De même certains discours sur le dépassement de soi et l'individualisme à outrance, introduit au cours des années quatre-vingt pour motiver le personnel, apparaissent aujourd'hui surannés, et les "stages en condition extrême ", imposés parfois à l'encadrement, disparaissent progressivement des catalogues des agences de voyages.

8 Si la cohésion et l'homogénéité dans l'entreprise rencontrent de nombreux adeptes, des voix discordantes se font également entendre. «L'univers managérial a pendant longtemps été dominé par l'idée d'une contribution positive de l'homogénéité et de la cohésion à l'efficacité de l'entreprise. Aujourd'hui, comme hier, beaucoup identifient plus ou moins consciemment ou explicitement l'homogénéisation et la cohésion, comme certains identifiaient dans le passé la vitalité d'un groupe social ou d'une société à son homogénéité génétique ou technique. » (Tapia, 1991).

\section{De l'influence des représentations sur le fonctionnement de l'entreprise}

9 La représentation constitue une "grille de lecture » qui contribue à construire la « réalité » de l'individu et façonne son comportement (Grize, Verges, Silem, 1987). Mais si cette grille de lecture intervient dans la construction du réel, elle agit comme un prisme déformant qui donne à chaque individu un sens différent aux mêmes objets ou événements.

10 Des représentations par trop hétérogènes peuvent engendrer des dysfonctionnements dans l'entreprise sous forme d'incompréhensions, d'incohérences, ou de dispersions d'efforts. En effet, le discours et son interprétation sont liés à l'univers social de l'individu, et la communication est d'autant plus aisée que "chacun a la même représentation de ce qui doit être dit et non-dit» (Orgogozo. 1988). Aussi, quand des membres du personnel ont des représentations trop contradictoires, le dialogue devient difficile voire conflictuel, et derrière une collaboration de façade se développent des stratégies d'acteurs où le non-dit prédomine et la rivalité se substitue à la coopération (Sainsaulieu. 1987). Cette divergence de vue est souvent la cause de malentendus car chaque individu est convaincu de la rationalité de ses choix et de la pertinence de ses analyses. De plus, confrontées les unes aux autres dans l'organisation, les représentations n'ont pas toujours tendance à estomper leurs différences. Elles se comportent plutôt comme un système de décodage qui ne retient que les informations n'entraînant pas de dissonance cognitive avec elle-même. Ainsi certains chefs de service seront persuadés que des employés présents en dehors des horaires de service sont là pour réaliser un travail personnel ou dérober du matériel et non. simplement, pour rattraper un retard (Pemartin, 1990). Inversement, certains employés ne verront que malice derrière le discours de l'encadrement. 
11 Mais la cohésion est-elle systématiquement bénéfique à l'organisation? Quand un groupe est uni, le moral de ses membres est généralement élevé mais cet avantage ne se traduit pas toujours en termes de performances (Fischer. 1990). Ainsi, à la suite des travaux d'Elton Mayo (1880-1949). certaines recherches ont montré que les groupes soudés établissent souvent leurs propres normes de productivité qui sont, le cas échéant, inférieures à celles qu'elles pourraient être (Schachter, 1951) ${ }^{1}$. De même, la cohésion peut générer un mode de fonctionnement où la recherche permanente du consensus neutralise l'esprit critique; le doute et les questions litigieuses étant alors évacués pour éviter l'affrontement. Janis $(1971,1982)$, a ainsi montré que les préoccupations unanimistes qui régnèrent dans les équipes gouvernementales des États-Unis à certains moments critiques de son histoire ont biaisé les décisions prises, l'attaque de Pearl Harbor en 1941, l'opération de la Baie des Cochons en 1961 ou l'affaire du Watergate au début des années soixante-dix.

Inversement, une faible cohésion peut donner lieu à des performances élevées comme l'ont révélé les travaux des chercheurs Hall et Watson (1970) qui réunirent plusieurs groupes d'individus, au cours d'une expérience, pour résoudre un problème de survie sur la lune. Les premiers avaient pour tâche d'aboutir à une solution collective, alors que les seconds avaient pour consigne de ne pas chercher le consensus. Ces derniers obtinrent de bien meilleurs résultats en faisant preuve d'une créativité sensiblement plus développée. De même, une recherche sur l'efficacité des directions d'entreprise aux États-Unis a montré que les groupes dirigeants hétérogènes s'avèrent généralement plus à même de faire face à des environnements turbulents que les groupes homogènes. En revanche ces derniers agissent, plus efficacement en période de stabilité (Murray, 1989).

En outre, les différences entre les membres d'une organisation enrichissent celle-ci d'opinions, de modes de pensée, et de compétences diverses. Elles élèvent le niveau d'objectivité des débats en abaissant le taux d'implication personnelle, favorise la tolérance entre les membres et améliore la réceptivité individuelle aux idées nouvelles (Tapia, 1991).

14 Afin de tenter d'évaluer l'influence des représentations sur le comportement des salariés, nous avons nous-même mené en 1993 une enquête quantitative de terrain dans des entreprises du secteur bancaire. Fondée sur les résultats d'un questionnaire renseigné par près d'un millier de personnes, celle-ci ne permis pas de déceler de nettes corrélations entre les représentations, le degré d'implication et l'efficacité individuels reconnue par l'organisation. Ce constat nous a conduit à ne pas retenir l'hypothèse communément admise à l'époque par de nombreux gestionnaires relative aux bienfaits supposés de l'homogénéité des représentations dans l'entreprise, même si une plus grande similarité représentationnelle fut observée au sein des équipes dirigeantes par rapport au reste du personnel.

15 Après d'autres travaux montrant que le discours volontariste exhortant à l'implication et au dépassement permanent ne suscite en général qu'une "adhésion de façade », il nous est apparu que l'influence des représentations sur le fonctionnement de l'entreprise ne pouvait être approché que localement par une démarche conjuguant les aspects sociocommunicationnel et technique des activités analysées. 


\section{Les représentations au cours de l'action collective}

16 Si la perspective théorique cognitive considère que le partage de croyances par les membres d'une organisation est quasiment nécessaire à l'action collective. (Schneider et Shrivastava, 1988, Seignourd. Dubois, 1999), K. E. Weick et les chercheurs de sa mouvance rejettent fermement cette hypothèse (1979) et n'accordent pas une grande importance à l'existence de représentations partagées. L'organisation ne serait pas le lieu d'une communauté d'idées, mais le point de rencontre quelque peu fortuit des intérêts de chacun, dans lequel les micro-comportements se transforment en macro-action organisationnelle. Le caractère partiel et précaire des consensus élaborés entre les individus conduit à concevoir les représentations collectives non comme un phénomène durable mais comme des points d'accord temporaires, négociés par les membres d'un groupe en interaction aussi appelés des "artefacts sociaux transitoires " (LangfieldSmith, 1992). Ainsi toute situation appelant une action collective n'imposerait pas le partage de valeurs ou de représentations communes mais seulement un «alignement » (Blumer. 1969). fondé sur un compromis propre à la situation (Weick. 1995 : 42-43). A l'instar des interactionnistes, K. E. Weick considère que le système d'actions organisées se construit grâce aux interactions au sein desquelles les individus inventent leur vie quotidienne (Koenig, 1996). Ces interactions s'inscrivent dans un cadre temporaire, fragile, qui est à reconstruire en permanence afin d'interpréter l'environnement. Cette vision de l'organisation, conçue comme un assemblage d'activités et d'interactions qui se développent entre ses membres, a été confortée par un certain nombre de recherches empiriques qui montrent que le sens au niveau collectif se focalise d'abord sur les moyens à mettre en œuvre puis, dans une moindre mesure, sur les objectifs à atteindre (Bourgeois, 1980 ; Porac, Thomas, Fuller, 1989). Les acteurs concernés pourraient donc travailler ensemble malgré l'existence de représentations et de buts différents. Pour prendre un exemple, à la pêche au saumon, les marins chargés de manœuvrer le filet du chalutier n'ont pas du tout la même représentation des différentes phases du travail, ni du découpage temporel de l'activité (Gatewood, 1985). Or ils mènent toutefois une action collective durable malgré cette absence de représentation partagée. La compréhension de telles situations de travail collectif passe par la recherche du « réseau complexe des représentations individuelles et des raisons poussant les individus à s'impliquer ", ainsi que par l'établissement du «schéma des codes servant à la prédiction réciproque des comportements » (Allaire et Firsirotu, $1988: 21$ ).

17 La prise en compte des résultats de travaux que nous avons récemment menés sur le terrain, concernant une activité de conception de système complexe, nous conduit cependant à relativiser ces conclusions. En effet, la conception du travail collectif proposée par E.W. Weik semble plus adaptée à une activité opérationnelle routinière, la pêche au saumon ou le suivi opérationnel d'une centrale nucléaire par exemple, qu'à une activité de conception. En effet, cette dernière se caractérise davantage par une coopération sur le plan des idées que sur celui des actions. L'action en conception consiste d'ailleurs principalement à formuler, traduire ou vérifier des idées énoncées. Aussi cette coopération particulière apparaît-elle difficile à établir sans un minimum de cohérence entre les objectifs individuels, même si chacun des acteurs de la conception est poussé par des motivations qui lui sont personnelles (curiosité intellectuelle, recherche de pouvoir, envie de reconnaissance, appât du gain...). L'objectif de réussite du projet en 
tant que satisfaction des exigences d'un client, en oubliant éventuellement le respect des coûts et délais qui pour certains apparaît secondaire, semble ainsi largement partagé. Cependant, cet accord sur un objectif général de réussite du projet ne signifie pas toujours une même compréhension des conditions de cette réussite.

Ainsi, contrairement à une activité opérationnelle, la conception ne peut suivre une procédure préétablie. La manière d'atteindre les objectifs fait l'objet de multiples confrontations et débats entre acteurs. Chaque action est négociée et conduit le plus souvent à une convergence représentationnelle locale et transitoire, même si des divergences d'approche conduisent parfois au blocage. En outre cette convergence des points de vue sur l'action est favorisée par l'existence de cadres normatifs et méthodologiques propres à chaque domaine d'activité.

\section{Conclusion}

19 L'homogénéité des représentations dans l'entreprise est souvent présentée comme un facteur de performance, voire une variable à manipuler au travers des politiques de ressources humaines et de communication interne. Mais si cette uniformité permet éventuellement d'apaiser les tensions et de limiter les conflits dans l'organisation, elle ne constitue pas, à nos yeux, un gage d'efficacité. En effet, la cohérence représentationnelle peut favoriser le dialogue et la capitalisation de l'expérience, mais également constituer un frein à l'innovation en limitant le champ des idées émises et en annihilant toute confrontation constructive.

Cependant l'analyse des pratiques sur le terrain, notamment en regard des théories sur l'interaction, montre l'influence des représentations des acteurs sur leurs actions. Celle-ci apparait beaucoup plus déterminante en conception, fondée sur la réflexion et la créativité, que lors d'activités opérationnelles, en grande partie régies par des procédures.

21 Aussi, contrairement à la vision de K. E. Weick pour qui l'action collective ne résulterait que d'accords occasionnels et limités, négociés par les membres d'un groupe en interaction aux intérêts multiples, la conception semble-t-elle imposer un accord minimal sur la définition même des objectifs pour engager l'action commune.

\section{BIBLIOGRAPHIE}

ALLAIRE Y., FIRSIROTU M.E., La culture organisationnelle : aspects théoriques, pratiques et méthodologiques. Montréal: Gaetan Morin, 1988, p. 3-48.

ALLPORT F.H.. A structuronomic conception of behavior: Individual and collective. Journal of Abnormal and Social Psychology, 1962, 64, p. 3-30.

ALTER N., Sociologie de l'entreprise et de l'innovation. Paris : PUF, 1996. 
BERNARD P.J., DAVIET J.-P. (sous la direction de), Culture d'entreprise et innovation. Paris : Presses du CNRS, 1992, $341 \mathrm{p}$.

BLUMER H., Symbolic interactionism: Perspective and method. New York: Englewood Cliffs. Practice Hall, 1969.

BOSCHE M., Corporate culture : la culture sans histoire. Revue Française de gestion, $\mathrm{n}^{\circ} 47-48$, sept. Oct.1984, p. 29-39.

BOUGON M.G., Congregate Cognitive Map: An Unified Dynamic Theory of Organization and Strategy. Journal of management Studies, 1992. n² 29, (3). p. 369-389.

BOURGEOIS L.J., Performance and consensus. Strategic Management Journal, 1980, vol. 1, p. 227-248.

CROZIER M. FRIEDBERG E., L'acteur et le système, les contraintes de l'action collective. Paris : Seuil, 1977. $436 \mathrm{p}$.

D'IRIBARNE P., La culture d'entreprise et sa prise en compte dans la gestion. Problèmes économiques, $\mathrm{n}^{\circ}$ 2000, 1986, p. 3-8.

FISCHER G.N., Les domaines de la psychologie sociale : le champ du social. Paris: Dunod, 1990, 276 p.

FORETT M. P., Creative Experience. New York: Longmans, Green, 1924.

GATEWOOD J.-B., Actions speak louder than words. In DOUGERTHY J.W.D. Direction in cognitive anthropology. Chicago: University of Illinois press, 1985, p. 199-219.

GRAY B., BOUGON M., DONNELLON A., Organizations as Constructions and Destructions of Meaning. Journal of management, 1985, vol. 11, n² 2, p. 83-98.

GRIZE J.-B., VERGES P., SILEM A., Salariés face aux nouvelles technologies. Vers une approche sociologique des représentations sociales. Paris : C.N.R.S., 1987,305 p.

HALL E.J., WATSON W.H.. The effects of normative intervention on group decision-making performance. Human relations 23, 1970. p. 299-317

HUTCHINS E., The social organization of distribued cognition. In RESNICK L.B., LEVINE J.-M., TEASLEY S.D. Perspectives on socially shared cognition. Washington, DC: American Psychological Association, 1991, p. 283-307.

JANIS I.L., Victims of Groupthink. Boston: Houghton Mifflin 1972. 280 p.

JANIS I.L., Groupthink: Psychological Studies of Policy Decisions and Fiascoes. Boston : Houghton Mifflin $1982,300 \mathrm{p}$.

KOENIG G.. WEICK K. L E. Revue française de gestion, 1996. n 108, p. 57-90.

LANGFIELD-SMITH K., Exploring the Need for a Shared Cognitive Map. Journal of Management Studies, 1992, n² 29 (3), p. 349-368.

LAROCHE H.. KARL E. WEICK (1995) Sensemaking in Organizations, Sage, Thousand Oaks. Californie. Sociologie du travail. 2/96, p. 225-232.

LE GOFF J-P.. Le travail comme vecteur d'identité. Panoramiques. I ${ }^{\text {er }}$ trimestre 1998. nº 32.

LEMAITRE N., La culture d'entreprise, facteur de performance. Revue Française de gestion, n 47-48, sept. 1984, p. 152-161.

MARCH J.-C. SIMON H.A., Les organisations, problèmes psychosociologiques. Paris : Dunod, 1981, 272 p. MARCH J.-G., Décisions et organisations. Paris : Editions Organisation, 1991. 
MURRAY A.I., Composition du groupe dirigeant et performance de l'entreprise. Revue Française de Gestion, juin, juil., août 1989, p. 6-26.

ORGOGOZO L, Les paradoxes de la communication. A l'écoute des différences. Paris : Ed. Organisation. 1988. $129 \mathrm{p}$.

OUCHI., Théorie Z : faire face au défi japonais. Paris : Interéditions. 1982.

PEMARTIN D., L'entreprise vue par ses salariés. Systèmes de valeurs, stéréotypes, mythes, rumeurs. Paris : Ed. Organisation, 1990, 223 p.

PETER T. WATERMAN R., Le prix de l'excellence. Paris: Interéditions, 1983.200 p.

PORAC J.. THOMAS H.. FULLER C.B.. Competitive Groups as Cognitive Communities: The Case of the Scottish Knitwear Manufacturers. Journal of Management Studies, 1989. n² 26, (4), p. 397-416.

SAINSAULIEU R., Sociologie de l'organisation et de l'entreprise, Paris : Dalloz, 1987. 390p.

SCHNEIDER SC. SHRIVASTAVA P.. Basic Assumptions Themes in Organizations. Human Relations, 1988, vol. 41. n 7. p. 493-516.

SEIGNOUR P., DUBOIS P.L., 1999, Les enjeux de marketing interne. Revue française de gestion, mars, avril, mai, p. 19-29.

SIMON H. A, Administration et processus de décision. Paris : Economica, 1983

SINGERY J. Représentations sociales et projets de changement technologique en entreprise. In ABRIC J.-C., Pratiques sociales et représentations. $2^{\mathrm{e}}$ édition. Paris : PUF, 1997, p. 179-216.

SUCHMAN L., Plans and situaled actions: The problem of human-machine communication. New York: Cambridge University Press. 1987.

TAPIA C, Management et sciences humaines. Paris : éditions d'Organisation Université, 1991, 188 p.

THEVENET M., Audit de la culture d'entreprise. Paris: Editions organisation, $1986-208$ p.

WEICK K. E., The Social Psychology of Organizing. Addison Westley: Reading, Mass., 1979 (2 édition). WEICK K.E., Sensemaking in Organizations. Sage Publications, Fondations for Organizational Science: Thousand Oaks, CA, 1995.

\section{NOTES}

1. Schachter et al.. An experimental study of cohesiveness and productiving - Human relations, août 1951. cité in J.P. Gruere, Management, aspect humains et organisationneh. Paris : PUF. 1991. p. 248-288.

\section{RÉSUMÉS}

Supposant à un courant de la pensée managériale très en vogue dans les années quatre-vingt, cet article réfute les bienfaits supposés de l'homogénéité du personnel dans l'entreprise. Il ne conteste pas l'influence des représentations sur l'action individuelle, mais s'interroge sur la 
cohérence de celles-ci dans l'action collective selon le type d'activité. Ainsi la conception collective implique-t-elle une certaine homogénéité des points de vue entre acteurs, concernant notamment la définition des objectifs à atteindre, alors que de simples accords occasionnels négociés par des individus en interaction, suffisent le plus souvent pour mener une activité opérationnelle de groupe.

Being opposed to a current of the managerial thought in vogue in the Eighties, this article refutes the supposed benefits of the homogeneity of the personnel in the company. It does not dispute the influence of the representations on the individual action, but wonders about the coherence of those in the collective action according to the type of activity. Thus the collective design implies a certain homogeneity front the points of view between actors, relating to the definition of the objectives in particular to he reached, whereas simple occasional agreements negotiated by individuals in interaction, are generally enough to undertake an operational activily of group.

\section{INDEX}

Mots-clés : représentation, culture d'entreprise, communication organisationnelle, conception, interaction

\section{AUTEUR}

\section{ARLETTE BOUZON}

Ariette Bouzon, Maître de conférences, habilitée à diriger des recherches, à l'Université de Toulouse III, au département Information-Communication. Chercheur au LERASS (Laboratoire d'Etudes et de Recherches appliquées en Sciences sociales), participe aux travaux de l'équipe de recherche MICS (Médiations en Information et Communication Spécialisées) et au groupe Org\&co portant sur la communication organisationnelle de la SFSIC. Thèmes de recherche : communication autour du risque, conception dans les organisations. 